\title{
Desempenho reprodutivo de vacas Nelore no Norte e Nordeste do Brasil ${ }^{1}$
}

\section{Danielle Maria Machado Ribeiro Azevêdo ${ }^{2}$, Raimundo Martins Filho ${ }^{3}$, Raimundo Nonato Braga Lôbo ${ }^{4}$, Carlos Henrique Mendes Malhado ${ }^{5}$, Raysildo Barbosa Lôbo ${ }^{6}$, Arlindo de Alencar Araripe Moura ${ }^{7}$, Edgard Cavalcanti Pimenta Filho ${ }^{8}$}

\author{
${ }^{1}$ Pesquisa realizada com recursos do CNPq/CAPES \\ 2 Embrapa Meio-Norte. \\ ${ }^{3}$ Bolsista CNPq/FAPEPIno DZO/CCA/UFPI. \\ ${ }^{4}$ Embrapa Caprinos. \\ 5 UESB. \\ 6 USP. \\ 7 UFC. \\ 8 UFPB.
}

RESUMO - Objetivou-se com este trabalho estimar os parâmetros genéticos e fenotípicos de características reprodutivas de fêmeas de rebanhos das regiões Norte e Nordeste do Brasil. As características estudadas foram idade ao primeiro parto (IPP), intervalo de partos (IDP), período de serviço (PS), número de serviços por concepção (NSC) e período de gestação (PG). Os modelos utilizados para cada característica foram selecionados por meio do procedimento MIXED do programa SAS e os componentes de variância foram estimados pelo método da Máxima Verossimilhança Restrita Livre de Derivada, utilizando-se

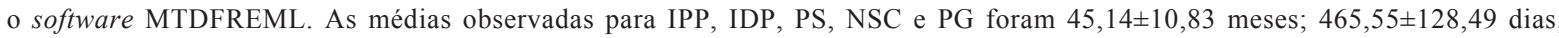
$165,76 \pm 110,29$ dias; $1,34 \pm 0,67$ serviços e $295,03 \pm 5,85$ dias, respectivamente. As estimativas de herdabilidade para IPP, IDP, PS, NSC e PG foram, respectivamente, $0,21 \pm 0,05 ; 0,05 \pm 0,02 ; 0,32 \pm 0,11 ; 0,05 \pm 0,04$ e $0,12 \pm 0,04$. As estimativas de repetibilidade encontradas para IDP, PS, NSC e PG foram, nesta ordem, 0,05;0,63;0,09 e 0,22. Os resultados sugerem que, apesar da pequena variabilidade genética para a maioria das características, a IPP e o PG são características reprodutivas que devem ser incluídas em programas de seleção. A elevada herdabilidade obtida para PS pode ser decorrente da estrutura dos dados analisados, provenientes, em sua grande maioria, de um mesmo rebanho. O baixo valor para a repetibilidade do IDP indica que o descarte de fêmeas com base no desempenho ao seu primeiro intervalo de partos pode ocasionar a eliminação de animais que poderão apresentar boa eficiência reprodutiva no futuro.

Palavras-chave: bovino de corte, eficiência reprodutiva, parâmetros genéticos, Zebu

\section{Reproductive performance of Nelore cows in the North and Northeast Regions of Brazil}

\begin{abstract}
The objective of this study was to estimate genetic and phenotypic parameters for reproductive traits of Nelore cows raised in the North and Northeast regions of Brazil. The traits studied were age at first calving (AFC), calving interval (CI), post-partum interval (PPI), number of services per conception (NSC) and gestation length (GL). The statistical analyses were done using the SAS program (Statistical Analysis System) and variance components were estimated by Restricted Maximum Likelihood method using the MTDFREML software. Means and heritability estimates for AFC, CI, PPI, NSC and GL were $45.14 \pm 10.83$ months; $465.55 \pm 128.49$ days; $165.76 \pm 110.29$ days; $1.34 \pm 0.67$ services and $295.03 \pm 5.85$ days and $0.21 \pm 0.05 ; 0.05 \pm 0.02 ; 0.32 \pm 0.11 ; 0.05 \pm 0.04$ e $0.12 \pm 0.04$, respectively. The repeatability for CI, PPI, NSC and GL were, respectively, $0.05 ; 0.63 ; 0.09$ and 0.22 . The results suggest that despite the small genetic variability for the reproductive traits, AFC and GL must be included in selection programs. The high heritability estimate for PPI is probably associated to the structure of the data, in its majority coming from the same herd. The repeatability estimate for CI suggests that female culling based on first calving interval is not accurate and there is a risk of culling animals with probable good reproductive efficiency.
\end{abstract}

Key Words: beef cattle, genetic parameters, reproductive efficiency, zebu cattle

\section{Introdução}

A baixa eficiência reprodutiva do rebanho bovino assume particular importância em regiões de clima tropical e subtropical visto que nestas a espécie Bos indicus representa a maior parte da população bovina. Neste contexto, a eficiência reprodutiva das fêmeas torna-se primordial, considerando-se que todo criador de bovinos de corte 
objetiva que cada uma de suas matrizes produza um bezerro saudável a cada ano, na tentativa de alcançar maior produtividade, competitividade e eficiência, otimizando a produção.

Nos trópicos, a idade ao primeiro parto (IPP) tardia é um dos principais fatores que afetam negativamente a produção de carne. Na raça Nelore, principal raça zebuína criada no Brasil, há grande variabilidade na IPP em diferentes rebanhos (47 meses - Biffani et al., 2000; 36 meses - Gunski et al., 2001), o que denota possibilidade de reduzi-la.

Em gado de corte, a duração do intervalo de partos (IDP) interfere diretamente na rentabilidade da exploração pecuária pois determina, em parte, o número de bezerros produzidos pela vaca e o intervalo de gerações, limitando a intensidade de seleção. Na raça Nelore, o IDP encontrado tem sido bastante variável, desde 358,7 dias (Oliveira Filho et al., 1991), próximo ao recomendado, de 12 a 13 meses, até 459,6 dias (Dias \& Oliveira, 1994).

O período de serviço (PS), um dos componentes do IDP, refere-se ao intervalo de uma parição à cobertura fértil subseqüente. Sua duração ideal é de 60 a 90 dias, considerando-se a meta de produção de um bezerro por ano. Entretanto, animais criados em regiões tropicais apresentam PS excessivamente longo. O período de gestação (PG), o outro componente do IDP, apesar de pouco variável em cada espécie, tem atraído a atenção de criadores e pesquisadores no sentido de selecionar animais para períodos de gestação mais curtos, a fim de proporcionar maior intervalo pós-parto até a próxima estação de acasalamento (Scarpati et al., 1998).

Outra característica importante na avaliação da eficiência reprodutiva de rebanhos, apesar de pouco comentada na literatura especializada, é o número de serviços por concepção (NSC). Esta característica apresenta alta variabilidade e embora o ideal seja apenas um serviço por gestação, na maioria dos rebanhos tropicais são necessários dois ou mais serviços, o que está relacionado, principalmente, ao manejo ao qual os animais são submetidos.

A herdabilidade das características reprodutivas geralmente é de baixa magnitude. Entre estas características, a IPP apresenta herdabilidade considerada de média a alta (0,46, Pereira et al. (1991); 0,29, Lôbo (1998), por sofrer influência direta da idade à puberdade, que, por sua vez, possui elevada herdabilidade. Deve ser ressaltado, no entanto, que estimativas de herdabilidade baixas também foram obtidas para IPP $(0,12$, Pereira et al., 2000). Para o IDP, a variação da herdabilidade entre rebanhos também é considerável-0,01 (Oliveira Filho et al., 1991) a 0,32 (Campello et al., 1999). Estimativas de herdabilidade para PS e NSC não foram encontradas na literatura revisada.
As características reprodutivas têm apresentado baixos valores de repetibilidade. Para o IDP, Schwengber et al. (2002) encontraram 0,09 como coeficiente de repetibilidade. Para PG, no entanto, Scarpati et al. (1998) estimaram a repetibilidade em 0,18. Para PS e NSC, não foram encontradas estimativas de repetibilidade na literatura consultada.

A raça Nelore, entre as raças zebuínas, tem grande preferência dos criadores das regiões Norte e Nordeste do Brasil. No entanto, informações sobre seu desempenho reprodutivo nestas regiões são escassas. Desta forma, objetivou-se com este estudo estimar e analisar os parâmetros genéticos de características reprodutivas de fêmeas Nelore criadas em rebanhos do Norte e Nordeste do Brasil, visando fornecer subsídios a programas de seleção e, conseqüentemente, incrementar a eficiência reprodutiva desses rebanhos.

\section{Material e Métodos}

Os dados analisados referem-se a 10.069 fêmeas da raça Nelore, nascidas entre 1971 e 2002, de rebanhos localizados nos estados da Bahia, do Maranhão, do Tocantins e do Pará (Tabela 1). Os rebanhos avaliados foram incluídos recentemente no Programa de Melhoramento Genético da Raça Nelore (PMGRN) e, portanto, o banco de dados analisado inclui informações anteriores à incorporação das técnicas de manejo recomendadas pelo PMGRN.

Os animais são criados a pasto e, na maioria dos rebanhos, touros e matrizes são mantidos juntos durante todo o ano, com diferentes proporções touro:vaca, dependendo do rebanho. Em algumas propriedades, no entanto, com manejo mais especializado, há monta controlada e inseminação artificial; destas propriedades, foram coletados os dados para período de gestação, período de serviço e número de serviços por concepção. Nos rebanhos avaliados, não há estação de monta.

As características estudadas foram idade ao primeiro parto (IPP), intervalo de partos (IDP), período de serviço (PS), número de serviços por concepção (NSC) e período de gestação (PG). Foram utilizadas observações de 3.937, 2.177, 962, 1.065 e 1.939 fêmeas, respectivamente, para IPP, IDP, PS, NSC e PG. Observações com valores extremos não compatíveis com a realidade biológica e com os intervalos verificados na literatura, assim como grupos contemporâneos com menos de três observações, foram excluídas. Os limites para IPP, IDP, PS e PG foram, respectivamente, inferior a 2.200 e variando de 321 a 863 , de 43 a 439 e de 264 a 306 dias. Após as restrições impostas, restaram 3.937, $6.473,1.993,2.239$ e 4.066 informações referentes às variáveis IPP, IDP, PS, NSC e PG. 
Para formação dos grupos contemporâneos, foram consideradas quatro estações de nascimento, definindo-se os trimestres assim: trimestre 1 - janeiro a março; trimestre 2 abril a junho; trimestre 3 - julho a setembro; e trimestre 4 outubro a dezembro. A idade da vaca ao parto, em dias, foi agrupada em seis classes: menor que 1.081 dias; de 1.081 a 1.426 ; de 1.427 a 1.785 ; de 1.786 a 2.145 ; de 2.146 a 3.586 dias

Tabela 1 - Fazendas participantes do PMGRN envolvidas na avaliação das características idade ao primeiro parto (IPP), intervalo de partos (IDP), período de serviço (PS), número de serviços por concepção (NSC) e período de gestação (PG), segundo o estado e o número de observações ( $n$ )

Table 1 - State of the PMGRN participant farm and respective number of records used in the evaluation of age at first calving (AFC), calving interval (Cl), post-partum interval (PPI), number of services per conception (NSC) and gestation length (GL)

\begin{tabular}{|c|c|c|c|c|}
\hline \multirow[t]{2}{*}{$\begin{array}{l}\text { Característica } \\
\text { Trait }\end{array}$} & \multirow[t]{2}{*}{$\begin{array}{l}\text { Código das fazendas } \\
\text { Code of the farms }\end{array}$} & \multirow[t]{2}{*}{$\begin{array}{c}\text { Estado } \\
\text { State }\end{array}$} & \multicolumn{2}{|c|}{$\begin{array}{c}\text { Observação } \\
\text { Record }\end{array}$} \\
\hline & & & $\mathrm{n}$ & $\begin{array}{l}\% \text { do total } \\
\% \text { of the total) }\end{array}$ \\
\hline \multirow[t]{11}{*}{ I P P } & 35 & BA & 176 & 4,47 \\
\hline & 36 & BA & 213 & 5,41 \\
\hline & 57 & MA & 142 & 3,61 \\
\hline & 74 & TO & 253 & 6,43 \\
\hline & 75 & TO & 1.022 & 25,96 \\
\hline & 81 & BA & 30 & 0,76 \\
\hline & 83 & BA & 182 & 4,62 \\
\hline & 85 & BA & 51 & 1,30 \\
\hline & 87 & BA & 30 & 0,76 \\
\hline & 88 & BA & 279 & 7,09 \\
\hline & 90 & PA & 1.559 & 39,60 \\
\hline \multirow[t]{12}{*}{ IDP } & 35 & BA & 527 & 8,14 \\
\hline & 36 & BA & 546 & 8,44 \\
\hline & 57 & MA & 84 & 1,30 \\
\hline & 74 & TO & 438 & 6,77 \\
\hline & 75 & TO & 937 & 14,48 \\
\hline & 81 & BA & 25 & 0,39 \\
\hline & 83 & BA & 931 & 14,38 \\
\hline & 85 & BA & 171 & 2,64 \\
\hline & 87 & BA & 55 & 0,85 \\
\hline & 88 & BA & 1.236 & 19,09 \\
\hline & 90 & PA & 1.510 & 23,33 \\
\hline & 95 & BA & 13 & 0,20 \\
\hline \multirow[t]{5}{*}{ PS } & 36 & BA & 252 & 12,64 \\
\hline & 75 & TO & 228 & 11,44 \\
\hline & 88 & BA & 122 & 6,12 \\
\hline & 90 & PA & 1.380 & 69,24 \\
\hline & 95 & BA & 11 & 0,55 \\
\hline \multirow[t]{6}{*}{ NSC } & 35 & BA & 108 & 4,62 \\
\hline & 36 & BA & 232 & 9,92 \\
\hline & 75 & TO & 257 & 10,99 \\
\hline & 88 & BA & 219 & 9,36 \\
\hline & 90 & PA & 1.510 & 64,56 \\
\hline & 95 & BA & 13 & 0,56 \\
\hline \multirow[t]{6}{*}{ PG } & 36 & BA & 394 & 9,69 \\
\hline & 75 & TO & 477 & 11,73 \\
\hline & 81 & BA & 14 & 0,34 \\
\hline & 88 & BA & 244 & 6,00 \\
\hline & 90 & PA & 2.893 & 71,15 \\
\hline & 95 & BA & 44 & 1,08 \\
\hline
\end{tabular}

e maior que 3.586 dias. Os grupos contemporâneos para análise das diferentes características foram assim formados: para IPP - fazenda, estação e ano de nascimento da vaca; para IDP, PS e NSC - fazenda, estação e ano de parto precedente; para PG - fazenda, ano e estação de acasalamento.

O procedimento MIXED do pacote estatístico SAS (Statistical Analysis System, 1999) foi utilizado para determinação do melhor modelo para cada característica estudada. Os componentes de variâncias necessários à obtenção dos parâmetros genéticos foram estimados pelo método de máxima verossimilhança restrita não derivativa (DFREML - DerivativeFree Restricted Maximum Likelihood), por meio de modelos animais com uma característica, pela aplicação do software MTDFREML (Multiple Trait Derivative-Free Restricted Maximum Likelihood), descrito por Boldman et al. (1995).

O modelo animal proposto para análise dos componentes de variância para a característica IPP continha o efeito aleatório do animal e efeitos fixos de grupo contemporâneo, da interação da variedade da vaca e do touro e sexo da cria, de acordo com o modelo misto: $\mathrm{Y}=\mathrm{X} \beta+\mathrm{Za}+\mathrm{e}$, em que: $\mathrm{Y}=$ vetor de observações da característica idade ao primeiro parto; $X=$ matriz de incidência dos efeitos fixos; $\beta=$ vetor dos efeitos fixos; $Z=$ matriz de incidência do efeito genético direto de cada animal; $a=$ vetor de efeitos genéticos diretos aleatórios; e = vetor de efeitos residuais aleatórios, assumindo-se as seguintes pressuposições: $y$, a e e possuem distribuição normal multivariada; $\mathrm{V}(\mathrm{a})=\mathrm{A} \sigma^{2}{ }_{\mathrm{a}} ; \mathrm{V}(\mathrm{e})=\mathrm{R}=\mathrm{I} \sigma^{2}{ }_{\mathrm{e}}$ $\operatorname{Cov}(\mathrm{a}, \mathrm{e})=0$.

O modelo geral para as características IDP, PG, PS e NSC consistiu de efeitos fixos e aleatórios. Os efeitos aleatórios foram o de animal e o de ambiente permanente, além do resíduo. Os efeitos fixos variaram de acordo com a característica: IDP - grupo contemporâneo, interação variedade da matriz e variedade do reprodutor, classe de idade da vaca e sexo da cria anterior; PG - grupo contemporâneo, interação variedade da matriz e variedade do reprodutor, classe de idade da vaca e sexo da cria; PS - grupo contemporâneo, interação variedade da matriz e variedade do reprodutor, classe de idade da vaca e tipo de acasalamento (monta controlada ou inseminação artificial); NSC - grupo contemporâneo, variedade e classe de idade da matriz.

\section{Resultados e Discussão}

A idade ao primeiro parto $(45,14 \pm 10,83$ meses) (Tabela 2$)$ condiz com a realidade dos rebanhos explorados em regiões tropicais, onde a alimentação é basicamente pastagem. O valor obtido foi superior aos encontrados (Biffani et al., 2000; De Los Reyes-Borjas et al., 2002) para a raça Nelore, em trabalhos realizados no Brasil, inclusive em artigo publicado 
Tabela 2 - Estatísticas descritivas das características reprodutivas estudadas em fêmeas Nelore criadas em rebanhos do Norte e Nordeste do Brasil

Table 2 - Statistics for reproductive traits of Nellore females raised in the North and Northeast regions of Brazil

\begin{tabular}{|c|c|c|c|c|c|}
\hline $\begin{array}{l}\text { Estatística } \\
\text { Statistic }\end{array}$ & $\begin{array}{l}\text { IPP (meses) } \\
A F C \text { (months) }\end{array}$ & $\begin{array}{c}\text { IDP (dias) } \\
C I \text { (days) }\end{array}$ & $\begin{array}{l}\text { PS (dias) } \\
\text { PPI (days) }\end{array}$ & $\begin{array}{l}\text { NSC (dias) } \\
\text { NSC (days) }\end{array}$ & $\begin{array}{l}\text { PG (dias) } \\
G L \text { (days) }\end{array}$ \\
\hline $\mathrm{N}^{0}$. de registros & 3.937 & 6.473 & 1.993 & 2.339 & 4.066 \\
\hline $\begin{array}{l}\text { Record number } \\
\text { Média }\end{array}$ & 45,14 & 465,55 & 165,76 & 1,34 & 295,03 \\
\hline $\begin{array}{l}\text { Average } \\
\text { DP } \\
S D\end{array}$ & 10,83 & 128,49 & 110,29 & 0,67 & 5,85 \\
\hline $\begin{array}{l}\text { CV (\%) } \\
\text { Mínimo }\end{array}$ & $\begin{array}{l}23,99 \\
21,29\end{array}$ & $\begin{array}{c}27,60 \\
321,00\end{array}$ & $\begin{array}{l}66,54 \\
43,00\end{array}$ & $\begin{array}{c}50,15 \\
1\end{array}$ & $\begin{array}{c}1,98 \\
264,00\end{array}$ \\
\hline Minimum & 73,00 & 863,0 & 439,00 & 6 & 306,00 \\
\hline
\end{tabular}

IPP = idade ao primeiro parto; IDP = intervalo de partos; PS = período de serviço; NSC = número de serviços por concepção; PG = período de gestação. $A F C=$ age at first calving; $\mathrm{Cl}=$ calving interval; $P P I=$ post-partum interval; $N S C=$ number of services per conception; $G L=$ gestation length .

com dados de rebanhos também participantes do PMGRN (Schwengber et al., 2002).

Na realidade, esta elevada idade ao primeiro parto (IPP) já era esperada, pois esta característica depende, em grande parte, de fatores ambientais, principalmente nutrição e manejo reprodutivo. Quanto à nutrição, deve-se salientar que um dos principais motivos para o aparecimento tardio da puberdade nos rebanhos zebuínos no Brasil é a estacionalidade da produção de forragens, associada à ausência de suplementação alimentar na primeira estação seca após o desmame, quando a fêmea ainda está em crescimento. Este fator deve ser observado com maior cuidado em rebanhos das regiões Norte e Nordeste do Brasil, como no caso deste trabalho, em virtude das peculiaridades edafoclimáticas dessas regiões, responsabilizadas em âmbito mundial por deficiências nutricionais não apenas de animais, mas também da população humana.

Quanto ao manejo reprodutivo, é importante observar a opção, por parte dos criadores, da época de entrada das novilhas em reprodução considerando apenas o peso ideal, em detrimento da idade, o que pode contribuir para que a média de IPP continue alta. Neste sentido, o PMGRN propõe, em alguns rebanhos participantes, a entrada das novilhas em reprodução entre 12 e 14 meses de idade, independentemente do peso, com o propósito de identificar e selecionar aquelas mais precoces. Nos rebanhos avaliados, a idade média ao primeiro parto $(45,14$ meses $)$ denota que as novilhas estão, provavelmente, sendo acasaladas pela primeira vez aproximadamente aos três anos de idade, o que é bastante tarde para o primeiro acasalamento.

A interação variedade da matriz*variedade do reprodutor influenciou significativamente $(\mathrm{P}<0,01)$ a IPP (Tabela 3). Fêmeas acasaladas com machos da variedade
Padrão tiveram menor IPP que fêmeas acasaladas com machos da variedade Nelore Mocho. Este fato deve-se provavelmente ao menor número de observações referentes a animais Nelore Mocho comparado ao número de observações em Nelore Padrão.

O sexo da cria teve efeito significativo $(\mathrm{P}<0,01)$ sobre a IPP. Quando as fêmeas pariram crias do sexo masculino, sua IPP foi superior à daquelas que pariram bezerras, provavelmente em razão da duração da gestação, mais longa em crias do sexo masculino $(\mathrm{P}<0,01)$, como observado também por Cavalcante et al. (2001b).

O valor estimado para a herdabilidade da $\operatorname{IPP}(0,21 \pm 0,05$; Tabela 4), próximo aos encontrados por Pereira et al. (2001), foi inferior aos obtidos por De Los Reyes-Borjas et al. (2002) e superior aos observados por Biffani et al. (2000) e Pereira et al. (2000), todos para a raça Nelore. A herdabilidade obtida, de magnitude moderada, sugere a existência de variabilidade genética aditiva suficiente para permitir a obtenção de ganhos genéticos a partir da seleção de animais mais precoces, mesmo que melhorias no manejo possam ser alternativas mais rápidas para a redução da IPP nestes rebanhos.

Pode-se inferir, portanto, que a redução da expressão da IPP na raça Nelore em rebanhos do Norte e Nordeste do Brasil, por meio da seleção, poderia trazer benefícios econômicos de forma mais gradual que as alterações de manejo, porém com tendência a ser duradoura, se proporcionado manejo mínimo adequado para fêmeas de recria. Desta forma, os ciclos reprodutivos se iniciariam mais cedo, acarretando maior longevidade em decorrência da precocidade e, como conseqüência, cada fêmea teria maior número de ciclos produtivos ao longo de sua vida, o que permitiria maior retorno do valor investido em manutenção no rebanho de recria. 
Tabela 3 - Médias por quadrados mínimos, erros-padrão e número de observações, por interação variedade da vaca*variedade do reprodutor, para as características idade ao primeiro parto (IPP), intervalo de partos (IDP) e período de serviço (PS) para fêmeas Nelore criadas no Norte e Nordeste do Brasil

Table 3 - Least square means, standard errors and number of records of the female *male interaction, for age at first calving (AFC), calving interval (CI) and post-partum interval (PPI) traits, in the North and Northeast regions of Brazil

\begin{tabular}{lccc}
\hline $\begin{array}{l}\text { Interação variedade da vaca*variedade do reprodutor } \\
\text { Interaction female*male* }\end{array}$ & $\begin{array}{c}\text { IPP (meses) } \\
A F C \text { (months) }\end{array}$ & $\begin{array}{c}\text { IDP (dias) } \\
C I \text { (days) }\end{array}$ & $\begin{array}{c}\text { PS (dias) } \\
P P I \text { (days) }\end{array}$ \\
\hline Padrão x Padrão & $45,17 \pm 0,21^{\mathrm{c}}(2.539)$ & $466,93 \pm 3,21^{\mathrm{a}}(4.216)$ & $159,67 \pm 8,79^{\mathrm{a}}(1.427)$ \\
Padrão x Mocho & $48,82 \pm 0,45^{\mathrm{a}}(371)$ & $448,18 \pm 8,49^{\mathrm{bc}}(267)$ & $133,35 \pm 16,20^{\mathrm{abc}}(74)$ \\
Mocho x Padrão & $45,39 \pm 0,55^{\mathrm{c}}(200)$ & $442,37 \pm 8,47^{\mathrm{c}}(257)$ & $98,76 \pm 17,41^{\mathrm{c}}(46)$ \\
Mocho x Mocho & $47,47 \pm 0,32^{\mathrm{b}}(827)$ & $460,43 \pm 4,74^{\mathrm{ab}}(1.733)$ & $146,75 \pm 10,38^{\mathrm{b}}(446)$ \\
\hline
\end{tabular}

* Médias com letras diferentes na mesma coluna diferem estatisticamente $(P<0,05)$

${ }^{*}$ Means with different letters in the same column differ $(P<0.05)$ statistically.

Tabela 4 - Componentes de variância e parâmetros genéticos e fenotípicos das características reprodutivas estudadas em fêmeas da raça Nelore criadas no Norte e Nordeste do Brasil

Table 4 - Variance components and genetic and phenotypic parameters for reproductive traits of Nellore females raised in the North and Northeast regions of Brazil

\begin{tabular}{|c|c|c|c|c|c|}
\hline $\begin{array}{l}\text { Característica } \\
\text { Trait }\end{array}$ & $\sigma_{a}^{2}$ & $\sigma_{\mathrm{ep}}^{2}$ & $\sigma_{\mathrm{e}}^{2}$ & $h^{2}$ & $\mathrm{r}$ \\
\hline IPP $(A F C)^{*}$ & $9.544,94$ & - & $35.832,28$ & $0,21 \pm 0,05$ & - \\
\hline IDP $(C I)$ & 683,90 & 0,006 & $13.387,65$ & $0,05 \pm 0,02$ & 0,05 \\
\hline PS (PPI) & $3.464,92$ & $3.315,85$ & $4.021,26$ & $0,32 \pm 0,11$ & 0,63 \\
\hline $\mathrm{NSC}(N S C)$ & 0,02 & 0,02 & 0,38 & $0,05 \pm 0,04$ & 0,09 \\
\hline $\mathrm{PG}(G L)$ & 3,46 & 3,03 & 23,61 & $0,12 \pm 0,04$ & 0,22 \\
\hline
\end{tabular}

* IPP = idade ao primeiro parto; IDP = intervalo de partos; PS = período de serviço; NSC = número de serviços por concepção; PG = período de gestação $\sigma^{2}=$ variância genética aditiva direta; $\sigma^{2}$ = variância de ambiente permanente; $\sigma^{2}=$ variância residual; $h^{2}=$ herdabilidade; $r=$ repetibilidade

${ }^{*} A F C=$ age at first calving; $C l=$ calving interval; $P P I=$ post-partum interval; $N S C=$ number of services perconception; $G L=$ gestation length; $\sigma^{2}{ }_{a}=$ additive genetic variance; $\sigma^{2}{ }_{e p}=$ permanent environment variance; $\sigma_{e}^{2}=$ residual variance; $h^{2}=$ heritability; $r=$ repeatability

O intervalo de partos (IDP) médio dos rebanhos incluídos nessa análise foi de 465,55 dias (Tabela 2), longe do valor ideal para obtenção de um bezerro ao ano. Valor semelhante foi obtido por Gonçalves et al. (1996) e valores superiores foram encontrados por Biffani et al. (2000) e De Los Reyes-Borjas et al. (2002). Outros autores (Campello et al., 1999; Cavalcante et al., 2000; Schwengber et al., 2002) relataram resultados inferiores.

A variação no manejo entre rebanhos, principalmente nutricional e reprodutivo, provavelmente foi responsável por grande parte das diferenças entre o resultado obtido neste trabalho e aqueles citados na literatura. Deve-se enfatizar que isso ocorre principalmente quando são comparados os resultados deste trabalho, obtidos em rebanhos localizados nas regiões Norte e Nordeste do país, com aqueles verificados em outras regiões mais favoráveis à pecuária ou com resultados encontrados a partir de dados coletados em maior número de estados do Brasil, em que os altos valores são diluídos, ocasionando menor média de IDP para o rebanho nacional. Neste sentido, ressalta-se que Schwengber et al. (2002), que obtiveram resultados inferiores aos deste trabalho, também avaliaram rebanhos do PMGRN, porém englobando informações de vários estados brasileiros.

A interação variedade da matriz*variedade do reprodutor influenciou significativamente $(\mathrm{P}<0,05)$ o IDP. Quando houve presença de Nelore Mocho na interação, fêmea ou reprodutor, ocorreram menores IDP (Tabela 3), provavelmente em virtude do menor tamanho das crias nascidas, visto que o Nelore Padrão tem maior tradição e seu processo seletivo para crescimento está mais adiantado. Assim, as crias resultantes de acasalamento Padrão x Mocho exigiriam menos de sua mãe $\mathrm{e}$, conseqüentemente, permitiriam que manifestassem estro mais cedo após o parto.

A classe de idade da vaca também exerceu influência significativa $(\mathrm{P}<0,05)$ sobre o IDP (Tabela 5). Fêmeas da primeira classe de idade apresentaram maior IDP que aquelas da segunda ou terceira classe de idade, corroborando o resultado obtido por Pereira et al. (2000), também para a raça Nelore. A maior duração do IDP em fêmeas de primeira classe de idade decorre de sua maior exigência, em termos nutricionais, visto que ainda não atingiram o seu desenvolvimento corporal completo. É importante ressaltar que o reduzido número de fêmeas na segunda classe de idade é ocasionado pelo descarte, em nível de propriedade, daquelas novilhas consideradas inferiores para reprodução ao final dos três anos de idade (primeira classe de idade), ficando na fazenda apenas aquelas consideradas as melhores do plantel. O aumento do número de fêmeas nas classes de idade subseqüentes é provocado pela incorporação de fêmeas no rebanho por meio da compra de animais adultos. 
Tabela 5 - Médias por quadrados mínimos, erros-padrão e número de observações, por classe de idade da vaca, para as características intervalo de partos (IDP), período de serviço (PS) e número de serviços por concepção (NSC), em dias, para fêmeas Nelore criadas no Norte e Nordeste do Brasil

Table 5 - Least square means, standard errors and number of records by female age class for calving interval (CI), post-partum interval (PPI) and number of services per conception (NSC), in days, of Nellore females raised in the North and Northeast regions of Brazil

\begin{tabular}{|c|c|c|c|}
\hline $\begin{array}{l}\text { Classe de idade da vaca* } \\
\text { Female age class }\end{array}$ & $\begin{array}{c}\mathrm{IDP}^{* *} \\
C I\end{array}$ & $\begin{array}{l}\text { PS } \\
P P I\end{array}$ & $\begin{array}{l}\mathrm{NSC} \\
N S C\end{array}$ \\
\hline 1 & $471,69 \pm 6,18^{\mathrm{a}}(1.026)$ & $115,32 \pm 30,86^{\mathrm{bc}}(12)$ & $1,17 \pm 0,17^{\text {abcd }}(17)$ \\
\hline 2 & $379,78 \pm 9,98^{\mathrm{c}}(207)$ & $42,06 \pm 15,16^{\mathrm{d}}(79)$ & $0,94 \pm 0,08^{\mathrm{d}}(102)$ \\
\hline 3 & $442,38 \pm 6,18^{b}(872)$ & $136,93 \pm 10,66^{\mathrm{b}}(415)$ & $1,13 \pm 0,05^{\mathrm{c}}(477)$ \\
\hline 4 & $480,86 \pm 5,48^{\mathrm{a}}(1.107)$ & $168,72 \pm 10,00^{\text {ac }}(598)$ & $1,20 \pm 0,04^{\mathrm{bc}}(636)$ \\
\hline 5 & $473,70 \pm 4,26^{\mathrm{a}}(2.157)$ & $176,93 \pm 8,78^{\mathrm{a}}(673)$ & $1,26 \pm 0,03^{\mathrm{ab}}(802)$ \\
\hline 6 & $478,46 \pm 4,94^{\mathrm{a}}(1.104)$ & $167,89 \pm 10,50^{\mathrm{ac}}(216)$ & $1,33 \pm 0,04^{\mathrm{a}}(305)$ \\
\hline
\end{tabular}

* Classe 1 - menor que 1.081 dias; classe 2 - entre 1.081 a 1.426 dias; classe 3 - de 1.427 a 1.785 dias; classe 4 - de 1.786 a 2.145 dias; classe 5 - de 2.146 a 3.586 e classe 6 - maior que 3.586 dias.

** Médias com letras diferentes na mesma coluna diferem estatisticamente $(P<0,05)$

* Class 1: lower than 1.080 days; class 2: 1.081-1.426 days; class 3: 1.427-1785 days; class 4:1780-2.145 days; class 5: 2.146-3.586 days and class 6: larger than 3.586 days

** Means with different letters in the same column differ $(P<0.05)$ statistically.

A herdabilidade do IDP obtida, $0,05 \pm 0,02$ (Tabela 4), foi próxima às encontradas por Biffani et al. (2000) e De Los Reyes-Borjas et al. (2002), de 0,08 e 0,07 , respectivamente, porém inferior ao resultado encontrado por Campello et al. (1999), de 0,32. A baixa herdabilidade nestes rebanhos indica pequena variabilidade genética aditiva na expressão do IDP, sendo esta característica mais influenciada pelas condições de ambiente. O IDP nestes rebanhos pode, portanto, ser mais fácil e rapidamente manipulado ao serem implementadas medidas de manejo mais adequadas.

A repetibilidade encontrada para o IDP foi de 0,05 (Tabela 4), implicando que a permanência ou não de fêmeas no rebanho de reprodução não deve ser pautada apenas no resultado obtido para o IDP, pois o valor obtido tem pequena probabilidade de se repetir ao longo da vida produtiva da matriz.

A duração média da gestação obtida neste estudo (295,03 dias) foi similar aos valores encontrados, para vacas Nelore, por Scarpati et al. (1998) e Schwengber et al. (2002), porém superior ao observado por Cavalcante et al. (2001b) - 284,7 dias. A seleção de fêmeas zebuínas considerando a duração da gestação tem sua importância caracterizada por possibilitar maior número de bezerros produzidos pela vaca durante sua vida produtiva, o que traria reflexos econômicos positivos, pois é bastante longo se comparado ao dos taurinos. A seleção para reduzir o período de gestação (PG) poderia, uma vez indicada, ser realizada utilizando-se reprodutores avaliados para menor duração de gestação. Neste sentido, Lôbo et al. (2002) sugerem a utilização de touros com DEP negativa, expressando os dias a menos de gestação. No entanto, deve-se ressaltar que períodos de gestação mais curtos estão ligados a menores pesos ao nascer e à gestação de bezerros do sexo feminino (Cavalcante et al., 2001b), como observado neste trabalho.
A herdabilidade estimada para PG, $0,12 \pm 0,04$ (Tabela 4), foi inferior às obtidas por Scarpati et al. (1998), Gunski et al. (2001), Pereira et al. (2002) e Schwengber et al. (2002) de, 0,$14 ; 0,33 ; 0,26$ e 0,15 , respectivamente, não tendo sido observado na literatura revisada valor inferior ao estimado. O valor obtido indica que, sendo satisfatório o desempenho de outras características de maior herdabilidade, seria viável a seleção direta para o PG, podendo ser selecionados reprodutores com base em sua DEP para menor duração da gestação. Assim, apesar de o PG apresentar pequeno CV (1,98\%; Tabela 2 ), o que pode dificultar a seleção, observa-se que existe variabilidade genética aditiva dentro da raça que deve ser explorada, visto que menor PG proporcionaria à vaca maior período de descanso até a próxima estação de monta. Neste contexto é importante frisar que esta seleção deve ser realizada apenas naqueles rebanhos em que o peso ao nascimento esteja dentro do ideal para a raça, evitando-se a perda de recém-nascidos por baixo peso ao nascer.

A repetibilidade encontrada para PG $(0,22)$ (Tabela 4) foi superior ao valor observado por Scarpati et al. (1998), 0,18 . Este valor de repetibilidade, que pode ser considerado moderado, indica uma considerável contribuição do ambiente permanente na variação desta característica, sendo possível estimar a duração das futuras gestações.

Operíodo de serviço obtido neste trabalho $(165,76 \pm 110,29$ dias) está bem acima do considerado ideal (60 a 90 dias) para produção de um bezerro/vaca/ano. Poucos são os trabalhos publicados que contemplam esta característica para rebanhos da raça Nelore (Miranda et al., 1982; Cavalcante et al., 2001a), com resultados variando de 149 a 239,56 dias. Isso decorre principalmente da utilização da monta natural como tipo de acasalamento de eleição nos rebanhos de corte no Brasil. O elevado valor encontrado neste estudo era esperado, visto que animais criados em regiões tropicais 
apresentam, comumente, PS excessivamente longo, em decorrência de fatores climáticos e práticas de manejo inadequadas, incluídas não apenas aquelas relacionadas às fêmeas, mas também aos touros na sua preparação para o acasalamento e à própria técnica de inseminação artificial.

Neste trabalho, os efeitos significativos $(\mathrm{P}<0,01)$ sobre o PS foram interação variedade da fêmea*variedade do reprodutor (Tabela 3 ) e classe de idade da matriz (Tabela 5). Fêmea acasalada com macho de igual variedade apresenta o maior PS, apesar de não ter ocorrido diferença significativa $(\mathrm{P}>0,05)$ entre o acasalamento de fêmeas Padrão com machos Mochos em relação aos demais acasalamentos (Tabela 3). Isto decorre, provavelmente, da estrutura dos dados, visto que há grande concentração de informações $(71,60 \%)$ no acasalamento de fêmeas e machos da variedade Padrão e apenas 2,30\% no acasalamento de fêmeas mochas com machos aspados.

Assim como para IDP, o menor tamanho da cria e sua conseqüente menor exigência em termos nutricionais podem estar relacionados à redução do PS quando da utilização de Nelore Mocho no acasalamento. Adicionalmente, fêmeas que parem crias menores, provavelmente, estão sujeitas a menor estresse ao parto e, conseqüentemente, têm menor predisposição à manifestação de distocias e complicações pós-parto, facilitando a involução uterina e a recuperação de seu eixo hipotalâmico-hipofisário-gonadal. Logo, a manifestação de estro pode ocorrer mais precocemente no pós-parto, possibilitando cobertura fértil também mais precoce.

As fêmeas da segunda classe de idade apresentaram o menor PS $(\mathrm{P}<0,01)$, seguidas pelas de $1^{\mathrm{a}} \mathrm{e}$ e ${ }^{\underline{a}}$ classes, que não diferiram entre si $(P>0,01)$. As fêmeas da $4{ }^{a}$ classe em diante apresentaram os maiores PS, apesar de os resultados obtidos para a 4 $\underline{\underline{a}}$ e $6 \underline{\text { a }}$ classes não terem diferido daqueles encontrados para a primeira classe, provavelmente em razão do pequeno número de informações nesta última classe (Tabela 5). Estes resultados podem ser explicados pelo fato de que as fêmeas da segunda classe já haviam atingido seu desenvolvimento completo e, portanto, podiam utilizar o alimento consumido apenas para mantença e restabelecimento do eixo endócrino, podendo entrar em estro mais cedo.

Contrariando as expectativas, os valores estimados para herdabilidade e repetibilidade do PS, respectivamente 0,32 e 0,63 , foram bastante elevados, o que, provavelmente é decorrente da estrutura dos dados analisados, visto que $71,02 \%$ desses dados foram provenientes de uma única propriedade. Desta forma, a variância ambiental pode ter sido subestimada, concorrendo para o aumento proporcional da variância genética aditiva e, conseqüentemente, da herdabilidade e da repetibilidade da característica. Em contrapartida, outra possibilidade é que, em decorrência da concentração de dados em uma única fazenda, pode ter ocorrido uma pequena conectabilidade entre esses dados, com diferenças genéticas marcantes entre os animais daquela fazenda em relação às demais, ocasionando aumento da variância genética.

Assim, optou-se por incluir os resultados de PS neste trabalho, por considerar-se muito pequeno o número de artigos publicados sobre características reprodutivas de rebanhos Nelore nas regiões Norte e Nordeste do Brasil, principalmente no que concerne à PS. Este fato pode ser demonstrado pela ausência de informações na literatura pesquisada sobre os coeficientes de herdabilidade e repetibilidade para esta característica na raça Nelore.

O número de serviços por concepção (NSC) obtido $(1,34)$ (Tabela 2) foi próximo do ideal (um serviço por concepção). Este resultado é melhor que o obtido por Oliveira Filho et al. (1986) (1,81), em inseminação artificial na raça Nelore.

Considerando-se que, para a maioria dos rebanhos criados em condições tropicais, são necessários dois ou mais serviços por concepção, em virtude das condições de manejo, alimentação e sanidade e de fatores inerentes ao próprio animal (Galina et al., 1994), pode-se inferir que os animais deste estudo apresentavam condições favoráveis para a fertilização.

Embora o valor obtido para NSC seja discordante em relação aos valores encontrados para PS e IDP, com os quais mantém íntima relação, deve-se atentar que o valor estimado para NSC foi resultado apenas do número de doses de sêmen utilizadas em IA ou do número de saltos em monta controlada em matrizes sob este manejo específico e não em todo o rebanho em monta a campo, do tipo de acasalamento predominante nos rebanhos avaliados, quando se torna difícil a mensuração desta característica. Assim, o manejo dos animais e o nível tecnológico das propriedades consideradas para avaliação do NSC parecem ser de qualidade superior, o que justificaria o reduzido NSC.

Fêmeas Nelore Mocho tiveram, em média, NSC inferior ao das fêmeas Padrão $(\mathrm{P}<0,01)$, o que sugere melhor fertilidade. A classe de idade da fêmea também exerceu influência significativa $(\mathrm{P}<0,01)$ sobre o NSC, tendo as fêmeas de segunda classe de idade o menor valor para esta característica. $\mathrm{O}$ pequeno número de informações referentes à primeira classe de idade deve-se à preferência de utilização de cobertura natural nas novilhas, o que impossibilita a observação do NSC (Tabela 5).

A herdabilidade estimada para NSC foi de $0,06 \mathrm{com}$ desvio-padrão de 0,05 . Este valor reduzido denota a pequena 
variância genética aditiva (Tabela 4) presente entre animais destes rebanhos. Desta forma, seriam os efeitos de meio que influenciariam, em maior grau, essa característica. A baixa repetibilidade obtida $(0,09)$ indica que outros parâmetros devem ser considerados quando do descarte destas fêmeas, evitando-se o descarte de uma boa reprodutora. Da mesma forma que para PS, a literatura pesquisada também não aborda estimativas para herdabilidade e repetibilidade para o NSC, o que decorre, provavelmente, da necessidade de observação da monta ou da inseminação para que seja contabilizada a informação, o que é impossível em criações extensivas utilizadas na maioria dos rebanhos de corte no Brasil.

\section{Conclusões}

O desempenho reprodutivo das fêmeas Nelore dos rebanhos avaliados no Norte e Nordeste do Brasil está aquém do ideal. Como os rebanhos são restritos a apenas duas regiões do Brasil, recomenda-se que novas avaliações sejam realizadas para esclarecimento das diferenças no desempenho reprodutivo entre as variedades Nelore Mocho e Nelore Padrão.

Apenas a idade ao primeiro parto e o período de gestação possuem considerável variabilidade genética e, conseqüentemente, maior possibilidade de terem suas médias melhoradas por seleção direta. Melhorias no manejo nutricional, sanitário e reprodutivo podem ter efeito mais rápido no incremento destas características.

\section{Literatura Citada}

BIFFANI, S.; MARTINS FILHO, R.; LUCIFERO, M. et al. Características reprodutivas de fêmeas da raça Nelore criadas na região Nordeste do Brasil. In: REUNIÃO ANUAL DA SOCIEDADE BRASILEIRA DE ZOOTECNIA, 37., 2000, Viçosa, MG. Anais... Viçosa, MG: Sociedade Brasileira de Zootecnia, 2000. p.202.

BOLDMAN, K.G.; KRIESE, L.A.; Van VLECK, L.D. et al. A manual for use of MTDFREML - a set of programs to obtain estimates of variances and covariances (DRAFT). Lincoln: USDA-ARS, 1995. 120p.

CAMPELlO, C.C.; MARTINS FILHO, R.; LOBO, R.N.B. Intervalo de partos e fertilidade real em vacas Nelore no estado do Maranhão. Revista Brasileira de Zootecnia, v.28, n.5, p.474-479, 1999.

CAVALCANTE, F.A.; MARTINS FILHO, R.; CAMPELLO, C.C. et al. Intervalo de partos em rebanho Nelore na Amazônia Oriental. Revista Brasileira de Zootecnia, v.29, n.5, p.13271331,2000

CAVAlCANTE, F.A.; MARTins FILHO, R.; CAMPEllo, C.C. et al. Período de serviço em rebanho Nelore na Amazônia Oriental. Revista Brasileira de Zootecnia, v.30, n.5, p.1456-1459, 2001a.

CAVALCANTE, F.A.; MARTINS FILHO, R.; CAMPELLO, C.C. et al. Período de gestação em rebanho Nelore na Amazônia
Oriental. Revista Brasileira de Zootecnia, v.30, n.5, p.1451$1455,2001 \mathrm{~b}$

DE LOS REYES-BORJAS, A.; MAGNABOSCO, C.U.; LÔBO, R.B et al. Estimativas de (co)variância e parâmetros genéticos para dias ao parto e características relacionadas em fêmeas Nelore. In: REUNIÃO ANUAL DA SOCIEDADE BRASILEIRA DE ZOOTECNIA, 39., 2002, Recife. Anais... Recife: Sociedade Brasileira de Zootecnia, 2002. (CD-ROM)

DIAS, F.M.G.N.; OLIVEIRA, H.N. Efeito da consangüinidade sobre o primeiro intervalo entre partos (IEP1) e a idade ao primeiro parto (IDADE1) de fêmeas da raça Nelore. In: REUNIÃO ANUAL DA SOCIEDADE BRASILEIRA DE ZOOTECNIA, 31. 1994, Maringá. Anais... Maringá: Sociedade Brasileira de Zootecnia, 1994. p.172.

GALINA, C.S.; ORIHUELA, A.; RUBIO, I. Behavioural characteristics of Zebu cattle with emphasis on reproductive efficiency. In: FIELDS, M.J.; SAND, R.S. (Eds.) Factors affecting calf crop. Florida: CRC Press, 1994. p.345-362.

GONÇALVES, J.N.S.; SCARPATI, M.T.V.; NARDON, R.F. Avaliação da fertilidade real e da capacidade mais provável de fertilidade real de matrizes de um rebanho da raça Nelore. In: REUNIÃO ANUAL DA SOCIEDADE BRASILEIRA DE ZOOTECNIA, 33. 1996, Fortaleza. Anais... Fortaleza: Sociedade Brasileira de Zootecnia, 1996. p.368-369.

GUNSKI, R.J.; GARNERO, A.V.; BEZERRA, L.A.F. et al. Idade ao primeiro parto, período de gestação e peso ao nascimento na raça Nelore. Ciência Agronômica, v.32, n.1/2, p.46-52, 2001.

LÔBO, R.B. Programa de melhoramento genético da raça Nelore. 2.ed. Ribeirão Preto: FINEP, 1994. 100p.

LÔBO, R.N.B. Genetic parameters for reproductive traits of zebu cows in the semi-arid region of Brazil. Livestock Production Science, v.55, p.245-248, 1998

LÔBO, R.B.; BEZERRA, L.A.F.; OLIVEIRA, H.N. et al. Avaliação genética de animais jovens, touros e matrizes. Ribeirão Preto: GEMAC/USP. 76p. 2002.

MIRANDA, J.J.F.; CARNEIRO, G.C.; FONSECA, C.G. et al. Fatores ambientes e genéticos relacionados com o intervalo entre partos na raça Nelore. Arquivo de Medicina Veterinária da UFMG, v.34, n.3, p.381-387, 1982 .

OLIVEIRA FILHO, E.B.; ZILLO, L.R.; DUARTE, F.A.M Fertilidade de vacas Nelore em relação ao sistema de acasalamento, número de serviços por concepção e número de crias por vaca. In: REUNIÃO ANUAL DA SOCIEDADE BRASILEIRA DE ZOOTECNIA, 23., 1986, Campo Grande. Anais... Campo Grande: Sociedade Brasileira de Zootecnia, 1986. p.361

OLIVEIRA FILHO, E.B.; ABREU, U.G.P.; BIANCHINI SOBRINHO, E. Avaliação genética quantitativa do intervalo entre partos em um rebanho Nelore, variedade pele rosa. In: REUNIÃO ANUAL DA SOCIEDADE BRASILEIRA DE ZOOTECNIA, 28., 1991, João Pessoa. Anais... João Pessoa: Sociedade Brasileira de Zootecnia, 1991. p.535.

PEREIRA, J.C.C.; AYALA, J.M.N.; OLIVEIRA, A.N. Efeitos genéticos e não genéticos sobre a idade ao primeiro parto e o intervalo entre partos de duas populações da raça Nelore. Arquivo Brasileiro de Medicina Veterinária e Zootecnia v.42, n.1, p.93-102, 1991.

PEREIRA, E.; ELER, J.P.; FERRAZ, J.B.S. et al. Correlação genética entre perímetro escrotal e algumas características reprodutivas na raça Nelore. Revista Brasileira de Zootecnia, v.29, n.6, p.1676-1683, 2000.

PEREIRA, E.; ELER, J.P.; FERRAZ, J.B.S. Análise genética de algumas características reprodutivas e suas relações com o desempenho ponderal na raça Nelore. Arquivo Brasileiro de Medicina Veterinária e Zootecnia, v.53, n.6, p.720-727, 2001.

PEREIRA, E.; ELER, J.P.; FERRAZ, J.B.S. Análise genética de características reprodutivas na raça Nelore. Pesquisa Agropecuária Brasileira, v.37, n.5, p.703-708, 2002. 
STATISTICAL ANALYSES SYSTEM - SAS. SAS/STAT ${ }^{\text {TM. SAS }}$ user's guide for windows environment. version 6.11. Cary: 1999.

SCARPATI, M.T.V.; LÔBO, R.B.; DE LOS REYES-BORJAS, A. et al. Modelos animais para estimação de parâmetros genéticos e fenotípicos do período de gestação na raça Nelore. In: REUNIÃO ANUAL DA SOCIEDADE BRASILEIRA DE ZOOTECNIA, 35. 1998, Botucatu. Anais... Botucatu: Sociedade Brasileira de Zootecnia, 1998. (CD-ROM)

SCHWENGBER, E.B.; LÔBO, R.B.; BEZERRA, L.A.F. Parâmetros genéticos da idade à primeira cria, intervalo de partos e período de gestação na raça Nelore. In: REUNIÃO ANUAL DA
SOCIEDADE BRASILEIRA DE ZOOTECNIA, 39., 2002, Recife. Anais... Recife: Sociedade Brasileira de Zootecnia, 2002. (CD-ROM).

Recebido: $16 / 03 / 05$ Aprovado: 24/02/06 